\title{
La barrière et le checkpoint : mise en politique de l'asymétrie
}

The barrier and the checkpoint: the politicisation of asymmetry

\section{Évelyne Ritaine}

\section{OpenEdition}

12 Journals

\section{Édition électronique}

URL : http://journals.openedition.org/conflits/17500

DOI : $10.4000 /$ conflits. 17500

ISSN : $1777-5345$

Éditeur :

CCLS - Centre d'études sur les conflits lilberté et sécurité, L'Harmattan

\section{Édition imprimée}

Date de publication : 30 mars 2009

Pagination : 15-33

ISBN : 978-2-296-07748-5

ISSN : 1157-996X

Référence électronique

Évelyne Ritaine, "La barrière et le checkpoint : mise en politique de l'asymétrie », Cultures \& Conflits [En ligne], 73 I printemps 2009, mis en ligne le 30 mars 2010, consulté le 30 mars 2021. URL : http:// journals.openedition.org/conflits/17500; DOI : https://doi.org/10.4000/conflits. 17500 


\title{
La barrière et le checkpoint : mise en politique de l'asymétrie
}

\author{
Evelyne RITAINE
}

Evelyne Ritaine est directrice de recherche FNSP (université de Bordeanx, Sciences Po Bordeaux, SPIRIT).

"L'ancien Big Brother était préoccupé par l'inclusion - l'intégration, mettre les gens en rang et les y maintenir. Ce qui intéresse le nouveau Big Brother, c'est l'exclusion - c'est chercher les gens qui ne conviennent pas au lieu où ils sont; les bannir de ce lieu et les déporter "là où est leur place"; ou, mieux encore, ne jamais les autoriser, pour commencer, à se rapprocher de ce lieu. Le nouveau Big Brother fournit aux officiers de l'immigration les listes de gens qu'ils ne devraient pas laisser entrer, et aux banquiers la liste de ceux qu'ils ne devraient pas admettre dans la compagnie de ceux qui sont dignes de crédit. Il donne aux gardes des instructions concernant ceux qu'ils devraient arrêter devant les grilles et ne pas laisser pénétrer dans la communauté de l'autre côté des grilles. Il insuffle aux surveillants du voisinage l'idée d'épier et de chasser les prétendus rôdeurs ou ceux qui ont des intentions louches - étrangers qui ne sont pas à leur place. Il offre aux propriétaires des circuits de télévision fermés, pour empêcher les indésirables de s'approcher. Il est le saint patron de tous les videurs, que ce soit au service d'une boîte de nuit ou d'un ministre d'Etat, ministre de l'Intérieur. » Zygmunt Bauman ${ }^{1}$

Paradoxe : dans notre univers de mobilité généralisée, l'érection de murs de 1 séparation est devenue une pratique fréquent, visant à empêcher la circulation des personnes ${ }^{2}$. La multiplication de ces murs de séparation ne peut

1. Bauman Z., Vies perdues. La modernité et ses exclus, Paris, Payot, 2006, pp. 242-243.

2. Cet article est issu d'une recherche en cours à SPIRIT (Sciences Po Bordeaux), développée en association avec le CERI (Sciences Po Paris). Je remercie particulièrement Christophe Jaffrelot (CERI) pour son soutien indéfectible et amical à ce projet. 
que laisser perplexe dans un monde défini comme un espace de flux et de risques globaux; dans un monde qui est aussi une société de contrôle, dominée par la logique des nouvelles technologies d'information, où «ce qui compte n'est pas la barrière, mais l'ordinateur qui repère la position de chacun, licite ou illicite, et opère une modulation universelle ${ }^{3}$ ".

Dans l'ère de l'espace, le territoire et son éventuelle fortification étaient garants de la sécurité collective, et leur contrôle constituait une prérogative régalienne majeure, définissant le pouvoir politique : celui des anciens empires (muraille de Chine, mur d'Hadrien), celui des villes et seigneuries du Moyen Age (fortifications), celui des Etats modernes (ligne Maginot et ligne Siegfried), celui des blocs militaires transnationaux (mur de l'Atlantique, mur de Berlin). L'intra-muros définissait une appartenance politique et une sûreté collective. Cette ère de l'espace, depuis longtemps minée par la globalisation, s'est définitivement close avec les attentats du 11 septembre 2001, révélation violente qu'il n'existe plus de refuge territorial :

« les lieux ne protègent plus, quand bien même on les a armés et fortifiés. Force et faiblesse, menace et sécurité sont à présent devenues, essentiellement, des questions extraterritoriales (et diffuses) qui échappent aux solutions territoriales (et centrées) ${ }^{4}$.»

L'espace global est tout entier devenu une «zone frontière », un espace fini et sous-régulé. C'est un espace de "vulnérabilité mutuelle assurée» dominé par l'incertitude, les coalitions fluctuantes, les actions de reconnaissance ${ }^{5}$. Dans cet espace incertain, les menaces - et l'anticipation des menaces - sont de nature asymétrique : le danger n'est plus seulement conventionnel et peut venir d'éléments difficilement identifiables (mafias, trafiquants, clans incontrôlés, terroristes, etc.). Un quartier urbain peut ne pas se sentir suffisamment sécurisé par les forces de l'ordre et chercher à organiser sa propre protection, en se blindant contre les intrusions. Un Etat peut juger insuffisant le contrôle conventionnel de son territoire par ses forces armées et de police, et choisir de renforcer le contrôle de son accès en blindant ses frontières pour prévenir des intrusions malveillantes. Le blindage contemporain de la limite territorialisée est une réponse asymétrique à la perception d'un péril asymétrique. Le mur n'est là que pour faire obstacle et permettre au checkpoint d'exercer pleinement sa fonction de contrôle : tous deux participent d'un dispositif de contrôle global qui s'exerce sur les déplacements des personnes ${ }^{6}$.

3. Deleuze G., "Post-scriptum sur les sociétés de contrôle ", Pourparlers 1972-1990, Paris, Editions de Minuit, 1990, http://infokiosques.net/imprimersans2.php3?id_article=214

4. Bauman Z., La Société assiégée, Rodez, Le Rouergue/Chambon, 2005, p. 127.

5. Ibid., p. 128.

6. Sur les différences entre société disciplinaire et société de contrôle, voir Foucault M., Surveiller et punir. Naissance de la prison, Paris, Gallimard, 1975 ; Foucault M., " La société disciplinaire en crise ", Dits et Ecrits, t. 2, Paris, Gallimard, 2001, pp. 532-534; Deleuze G., Pourparlers, 
Les murs de séparation contemporains peuvent ne pas sembler comparables dans leurs caractéristiques. Ils sont en effet dotés de technologies de blindage plus ou moins élaborées, allant de simples rangées de barbelés électrifiés 7 aux murs de béton, puis aux ouvrages équipés des technologies de surveillance les plus récentes. Ils ont aussi des caractères juridiques et politiques différents.

\section{Figure 1 - Murs contemporains}

\begin{tabular}{|c|c|}
\hline $\begin{array}{l}\text { Frontières } \\
\text { juridiques }\end{array}$ & Frontières blindées \\
\hline & $\begin{array}{l}\text { Etats-Unis / Mexique } \\
\text { Union européenne / Afrique } \\
\text { (Espagne / Maroc) } \\
\text { Inde / Pakistan } \\
\text { Inde / Bangladesh } \\
\text { Thailande / Malaisie } \\
\text { Chine / Corée Nord } \\
\text { Turquie / Irak } \\
\text { Arabie Saoudite / Irak } \\
\text { Arabie Saoudite / Yémen } \\
\text { Émirats / Sultanat Oman } \\
\text { Botswana / Zimbabwe }\end{array}$ \\
\hline $\begin{array}{l}\text { Quartiers } \\
\text { sociaux }\end{array}$ & $\begin{array}{l}\text { Murs de séparation } \\
\text { socio-urbains }\end{array}$ \\
\hline & $\begin{array}{l}\text { Gated communities } \\
\text { Enclaves urbaines fortifiées } \\
\text { Blindage zones touristiques }\end{array}$ \\
\hline
\end{tabular}

op. cit. ; Mattelart A. (entretien avec), «Société de la connaissance, société de l'information, société de contrôle », Cultures E Conflits, n64, hiver 2006, pp. 167-183, http://www.libertysecurity.org/article1044.html

7. Sur l'histoire politique du barbelé, voir Razac O., Histoire politique du barbelé. La prairie, la tranchée, le camp, Paris, La Fabrique, 2000. 
Les murs de séparation les plus fréquents, les cas de blindage de frontière, relèvent d'un sécuritaire civil et militaire : dans un amalgame troublant, ils sont censés protéger un territoire national du terrorisme, des trafics, de l'immigration clandestine ${ }^{8}$. Il s'agit d'un exercice de souveraineté étatique, puisque la barrière est construite sur le territoire de l'Etat décideur, dans un contexte de rapport de forces très déséquilibré avec le territoire voisin. Des milliers de kilomètres de frontière blindée s'étendent ainsi aujourd'hui entre les Etats-Unis et le Mexique, entre l'Union européenne et l'Afrique (notamment entre l'Espagne et le Maroc, mais aussi tout le long des côtes méditerranéennes par une forme de blindage maritime, une «mer blindée»), entre l'Inde et le Bangladesh, entre la Chine et la Corée du Nord, entre le Botswana et le Zimbabwe, entre l'Arabie Saoudite et l'Irak, etc.

D'autres cas relèvent d'un sécuritaire directement militaire : il s'agit de lignes de cessez-le-feu dont le tracé et la valeur juridique sont contestés par l'un ou l'autre des protagonistes. Imposer, par l'importance des dispositifs construits, des limites territoriales de fait semble bien être la stratégie sousjacente à l'érection de ces murs : ainsi de la construction par Israël d'un mur, et de nombreux dispositifs d'empêchement, sur et au-delà de la ligne verte de 1949, en Cisjordanie ; ainsi de la construction, par le Maroc, de murs successifs, sur et au-delà de la limite internationalement reconnue des Territoires sahraouis, au Sahara occidental; ainsi du blindage, par l'Inde, de la ligne de cessez-le-feu au Cachemire, qui semble entériner la partition, au grand dam $\mathrm{du}$ Pakistan et des nationalistes cachemiris. Dans ces cas, contrairement aux autres, il y a eu déplacement ou séparation des populations. Du sécuritaire militaire relèvent aussi les murs de séparation entre communautés, érigés dans des contextes de guerre civile par des forces militaires tierces, pour séparer les belligérants : les peacelines de Belfast entre quartiers catholiques et quartiers protestants, construits par l'armée britannique; les Bremer walls à Bagdad entre quartiers sunnites et quartiers chiites, placés par l’armée américaine.

Bien qu'apparemment éloigné de la logique qui préside aux cas précédents, le sécuritaire social atteste d'une logique de séparation homologue, à l'œuvre dans les enclaves urbaines fortifiées (comme les barrios privados d'Amérique latine), dans la construction de barrières préventives en milieu urbain ${ }^{9}$, et dans les gated communities, désormais répandues dans le monde entier. Ces dispositifs de séparation urbains supposent en effet l'anticipation permanente d'une

8. Didier Bigo relève cet amalgame dans plusieurs de ses travaux. Voir entre autres «Sécurité et immigration : vers une gouvernementalité par l'inquiétude ", Cultures $\&$ Conflits, n³1-32, 1998, http://www.conflits.org/index537.html

9. Comme dans les cas bien connus de Cuincy (France) : voir Belmessous H., «Voyage à travers les forteresses des riches ", Le Monde diplomatique, novembre 2002 ; de Padoue (Italie) : voir Aloïse S., « Padoue érige un mur pour isoler un ghetto d'immigrés », Le Monde, 16 août 2006. Cette barrière y est décrite comme "une succession de plaques d'acier bien enfoncées dans le sol avec une seule entrée surveillée jour et nuit par des policiers». 
menace extérieure, qui appelle le déploiement de techniques d'inspiration policière et militaire pour contrôler un territoire privatisé. Au sein de ce territoire, un mode de gouvernance privée tend à se substituer au gouvernement public.

Ces dispositifs de contrôle affichent donc toujours une fonction sécuritaire, dont les fins explicites - civiles, militaires ou sociales - sont différentes, quoique souvent emboîtées. Ils sont susceptibles d'une analyse spécifique à chacun, celle d'une sociologie politique des frontières, celle d'une géopolitique des contestations territoriales, celle d'une sociologie de la ségrégation urbaine. Cependant, peut-être ont-ils en commun une fonction de séparation politique inédite et révélatrice des tensions contemporaines dans le contexte de la globalisation. En les considérant tous comme des processus de blindage d'une limite territorialisée, destinés à contrôler la circulation des personnes, que peut-on apprendre du sens politique de ce geste de séparation, de ce Mur politique 10 ? Une des façons de réfléchir à ce sens politique, bien que non exclusive ${ }^{11}$, est de considérer le Mur comme une mise en politique de l'asymétrie et d'en décliner toutes les conséquences.

Les murs contemporains n'ont pas pour ambition de stopper des invasions ennemies, comme c'était le cas pour les fortifications anciennes. Ces murs ne prétendent pas empêcher les ressortissants de l'intérieur de s'enfuir, comme le faisait le mur de Berlin. Ces murs se préoccupent d'«enfermer dehors» les indésirables. En ce sens, le Mur, dont l'efficacité pratique peut toujours être discutée, est politiquement et symboliquement décisif : alors que la frontière ne désigne que l'étranger (symétriquement des deux côtés), alors que la ligne de cessez-le-feu est tracée d'un commun accord entre belligérants (symétriquement des deux côtés), alors que la différenciation entre quartiers obéit à une logique sociologique (symétriquement des deux côtés), le Mur fabrique, asymétriquement, le suspect. C'est cette asymétrie fondamentale, révélée par le caractère unilatéral de la décision d'érection et par les effets arbitraires de l'exclusion, qui caractérise la politique du Mur ${ }^{12}$.

Dans tous les cas, le Mur crée un nouvel espace public, asymétrique. Il définit un extérieur et un intérieur équivalents d'un espace du risque et d'un

10. Dans la suite du texte, l'emploi de la majuscule (le « Mur») désigne la politique du Mur, et donc l'ensemble du dispositif.

11. Il faut aussi s'interroger sur les conditions de prises de décision. Je développe dans un autre texte les enjeux de politique interne, notamment en termes de politiques identitaires, qui soustendent les prises de décisions qui constituent la politique du Mur; voir Ritaine E., " "Enfermer dehors" : les murs de séparation, une politique intérieure », à paraître, 2009.

12. D’après le Robert : "Symétrie : correspondance exacte en forme, taille et position de parties opposées; Dissymétrie : défaut de symétrie; Asymétrie : absence de symétrie». En matière stratégique, «le concept d'asymétrie doit être distingué de celui de dissymétrie : ce dernier indique une différence quantitative entre les forces ou la puissance des belligérants [...]. L'asymétrie en revanche souligne les différences qualitatives dans les moyens employés, dans le style et dans les valeurs des nouveaux ennemis ", in Bishara M., "Guerre totale contre un péril diffus. L'ère des conflits asymétriques", Le Monde diplomatique, octobre 2001, http://www.monde-diplomatique.fr 
espace de la protection. Il définit une communauté sociale et territoriale digne de protection : il est identitaire. Il définit des catégories dangereuses dont il convient de se protéger : il est exclusif, stigmatisant et humiliant. Il définit la légalité : la séparation provoque la transgression, infraction dans le franchissement non autorisé, clandestinité dans le séjour irrégulier. Dans tous les cas, la stratégie du Mur désigne un pouvoir, au sens foucaldien du terme de «mode d'action sur des actions des autres 13 » : celui qui décide ce qui est intérieur et ce qui est extérieur ; celui qui décide de la liberté de circulation des autres. Il marque un pouvoir de décision (décider de la séparation), un pouvoir de contrôle (contrôler les déplacements des autres), un pouvoir de catégorisation (légitimer la séparation par la stigmatisation des outsiders).

\section{«Etant donné un mur, que se passe-t-il derrière ? 14 » : une défiance asymétrique}

On ne construit jamais une barrière face à une puissance équivalente, ou à une partie considérée comme politiquement ou socialement respectable. Si l'autre partie est considérée comme fiable, le contrôle de la limite s'effectue de façon bilatérale : les Etats-Unis, par exemple, collaborent avec le Canada pour le contrôle de leur frontière nord, mais imposent une barrière à la frontière avec le Mexique (et donc avec l'Amérique du Sud en général). Le Mur court toujours le long d'une ligne de déséquilibre, ligne de faille de la globalisation, déséquilibre de richesse, déséquilibre de puissance.

Les cas de blindage de frontière désignent ainsi le plus souvent une forte dissymétrie économique : les écarts de revenus entre les Etats-Unis et le Mexique, l'Espagne et le Maroc (l'Union européenne et l'Afrique), la Grèce/l'Italie et les Balkans sont parmi les plus importants dans le monde ${ }^{15}$. Dans d'autres cas, le déséquilibre, aussi bien politique qu'économique, tient plus à une situation locale. Ainsi, en Cisjordanie et à Gaza, le Mur sépare-t-il un Etat riche et puissamment armé d'un territoire sinistré et sans réelle souveraineté politique ${ }^{16}$. Ainsi, entre le Botswana et le Zimbabwe, une barrière prétend-elle protéger le premier territoire des effets du collapsus économique et social du second. Ainsi en est-il de l'Inde, en pleine expansion économique, qui dresse une barrière entre

13. Foucault M., «Deux essais sur le sujet et le pouvoir », in Dreyfus H., Rabinow P., Michel Foucault, un parcours philosophique, Paris, Gallimard, 1984, pp. 296-321.

14. Tardieu J., Le professeur Froeppel, Paris, Gallimard, coll. « L'Imaginaire », 2003.

15 . «Les frontières les plus agitées sont celles qui séparent les populations dont les écarts de revenus sont les plus larges. On compte à l'heure actuelle quatre points chauds de ce genre : les frontières entre les Etats-Unis et le Mexique, l'Espagne et le Maroc, la Grèce (et l'Italie) et le sud des Balkans, l'Indonésie et Singapour (ou la Malaisie). L'écart de revenus est de plus de 7 pour 1 dans le dernier cas, de 4,5 pour 1 entre l'Espagne et le Maroc, de 4,3 pour 1 entre les Etats-Unis et le Mexique, et de 4 pour 1 entre la Grèce et l'Albanie », in Milanovic B., "Le véritable ennemi mortel de la mondialisation ", Le Monde, 12 septembre 2006 ; Branko M., Worlds Apart. Measuring International and Global Inequality, Princeton, Oxford, Princeton University Press, 2005.

16. L'écart de revenus entre Israël et les Territoires palestiniens est estimé de 20 à 1. 
son territoire et l'instabilité (économique, politique, religieuse, environnementale) de son voisin, le Bangladesh. Le déséquilibre peut aussi tenir à l'anarchie sociale provoquée par la guerre, comme en Irak actuellement, dont les voisins ont blindé leurs frontières (Arabie Saoudite, Turquie). Enfin, dans les gated communities, le Mur enserre des groupes socialement homogènes, ainsi séparés de ceux qui sont autres (des pauvres, des marginaux certes, mais aussi des groupes sociaux différents). Entre les deux côtés du Mur, il y a toujours un important déséquilibre de potentiel, ainsi qu'une défiance asymétrique.

La décision d'ériger le Mur exprime l'asymétrie, car elle ne dresse pas une barrière face à une partie équivalente, un autre Etat ou un autre groupe social, mais face à des risques d'intrusion de groupes peu identifiables : elle fonctionne à l'anticipation de la menace asymétrique. Elle est donc toujours unilatérale : elle dénie toute possibilité de négociation avec une partie équivalente, quand elle ne cherche pas à nier l'existence même d'une autre partie, comme dans les stratégies israélienne et marocaine. Elle émane toujours de l'acteur puissant : elle signe un arbitraire et le déséquilibre - voire l'absence - de l'échange politique ou de l'échange social. Aussi le Mur est-il toujours contesté, même quand il est érigé sur une frontière officielle ou sur une propriété privée. L'autre côté considère toujours que, si politique de sécurisation il doit y avoir, elle devrait être bilatérale. Le Mexique, par exemple, a violemment protesté contre le blindage de la frontière qui lui a été imposé par les Etats-Unis : "C'est la coopération, et non pas des murs, qui peuvent assurer la sécurité frontalière : il s'agit de notre frontière commune 17 ».

Lorsqu'il évoque les "guerres asymétriques» du monde globalisé, Zygmunt Bauman analyse l'asymétrie en deux sens. Le premier, classique en matière stratégique, est celui de guerres menées entre acteurs étatiques et acteurs non étatiques, dotés de moyens d'action qualitativement différents. Le second réfère aux effets de la globalisation sur les parties en conflit : d'un côté une élite globale, extraterritoriale et mobile, caractérisée par l'aisance et la vitesse de ses échanges; de l'autre, les dominés, «clonés au sol », empêchés d'aller et venir, aussi bien par manque de moyens matériels que par contraintes juridiques. La lutte de ceux qui contestent ce confinement est alors de tenter d'atteindre malgré tout l'élite globale dans ses déplacements et dans ses sanctuaires, ou du moins d'en montrer la vulnérabilité par des moyens non conventionnels (non-violence, guerre de l'information, violence politique, sabotages, guérillas, actes terroristes). Une des tentations des puissances dominantes face à la fluidité des réseaux adverses est alors de vouloir re-territorialiser ces phénomènes : désignation d'Etats voyous, rétention des suspects de terrorisme, blindage des zones de passage (militarisation des frontières, bou-

17. Ernesto Derbez, ministre mexicain des Relations extérieures, cité dans Paranagua P.A., «Le ministre des Affaires étrangères mexicain s'insurge contre le projet de mur américain ", Le Monde, 11 octobre 2006. 
clage des zones de transit) ${ }^{18}$. Dans cette conception de l'asymétrie, l'acteur territorialisé et dominant en termes de moyens conventionnels peut, en prétendant prévenir des risques perçus ou supposés, choisir de sécuriser son territoire en excluant non pas des adversaires mais tous les suspects potentiels.

L'ambiguïté politique du Mur est de reposer sur une anticipation de la menace au sein même des sociétés civiles : il n'y a pas deux protagonistes identifiés, mais un acteur territorialisé (Etat ou communauté locale) d'un côté, et toute une population suspectée de l'autre. Une décision étrangère a ainsi des conséquences majeures sur des personnes et des populations qui n'ont plus accès à une pleine souveraineté : les voyageurs qui n’ont pas le «bon passeport », les Palestiniens ou les Sahraouis sans Etat, les travailleurs migrants, les pauvres et les marginaux. Comme le disait le ministre de la Sécurité israélien: "[Il fallait] réfléchir au nombre de travailleurs palestiniens illégaux qui entraient en Israël depuis la Cisjordanie, même en cas de bouclage. Car $99 \%$ d'entre eux ne venaient que pour travailler, mais $1 \%$ pouvaient être des terroristes 19 ». Exactement comme dans les gated communities, certains choisissent de se séparer, et d'autres sont séparés de fait. L'arbitraire matérialisé par la présence du Mur est bien enregistré dans le langage, puisque le Mur n'est jamais nommé de la même façon des deux côtés : «barrière de sécurité » en Israël, «mur d'annexion» ou «mur d'apartheid» pour les Palestiniens; « mur de sécurité » au Maroc, «mur de la honte » pour les Sahraouis ; «border fence » aux Etats-Unis, «linea fatale » ou «il muro » pour les Mexicains, etc.

Cette décision arbitraire se fonde sur une stratégie préventive qui, en prétendant blinder un espace, retrouve un geste militaire. L'emphase mise sur l'importance des coûts financiers et sur le raffinement des technologies de surveillance est l'image la plus explicite de ce déséquilibre. Que l'on soit dans une situation militaire, dans une situation civile, ou dans une situation mêlant civil et militaire, les institutions militaro-industrielles (sociétés d'expertise en matière de défense, entreprises spécialisées dans les technologies d'information et de surveillance) sont présentes pour assurer la reconversion de technologies employées par les militaires : le Mur marque toujours une convergence du civil et du militaire, une «militarisation de la contiguitté 20 ».

\section{«La militarisation de la contiguïté » : une asymétrie technologique}

Il y a des enjeux de pouvoir dans la volonté d'imposer de telles pratiques de contrôle d'un territoire, des pratiques de blindage : en effet, les enjeux de la

18. Bauman Z., La Société assiégée, op. cit.

19. Avi Dichter, ministre de la Sécurité, 7 juillet 2006, entretien avec René Backmann, Un mur en Palestine, Paris, Fayard, 2006, p. 71.

20. Dal Lago A., "Note sulla militarizzazione delle contiguità », Conflitti Globali, n², 2005, http://www.libertysecurity.org/IMG/pdf_conflitti2.pdf 
«securitization» (l'acte de classification, le speech act, qui fait considérer qu'un problème relève de pratiques sécuritaires) vont bien au-delà des questions de sécurisation ${ }^{21}$. Le scénario politique de securitization des limites territoriales se déroule en trois actes : il s'agit d'abord de construire la contiguité comme risque, comme enjeu sécuritaire; il s'agit ensuite de trouver un certain type de solution sécuritaire, ici l'érection de barrières et l'installation d'un dispositif de contrôle des déplacements; il faut enfin élaborer une légitimation globale de cette entreprise politique ${ }^{22}$. Il peut ainsi y avoir tactique sécuritaire par la séparation civile : ce serait le cas dans les différentes formes de séparation urbaine. Il peut y avoir tactique sécuritaire par contrôle du limes : ce serait le cas des frontières blindées. Il peut y avoir tactique sécuritaire par déni de légitimité territoriale : ce serait le cas des lignes de cessez-le-feu outrepassées. Le Mur, en supposant l'existence d'un danger extérieur, instaure ainsi un étonnant contexte de défiance au sein même de la société civile. En effet, il fonctionne sur le double principe de la dissuasion et du contrôle, de l'obstacle et du triage.

Les gated communities et les barrios privados eux-mêmes, loin d'être de simples solutions architecturales, révèlent un dispositif de défense du territoire d'inspiration militaire. Pour l'urbanisme défensif, en effet :

«police, criminologues, urbanistes et architectes ont développé un ensemble de tactiques de conception architecturale pour aider à réduire et à contrôler la criminalité. Considérées dans leur ensemble, ces mesures de sécurité sont appelées "prévention de la criminalité par la conception environnementale" (Crime Prevention through Environmental Design, CPTED). Elles vont des changements de design architectural qui encouragent ou facilitent des comportements sociaux dissuasifs, à des mesures de "renforcement des cibles" (terme militaire employé pour le blindage des zones vulnérables), qui utilisent des barrières matérielles et des technologies de surveillance. Le CPTED emploie souvent les verrous, obstacles, barrières, barbelés, grilles et gardes de la prévention du crime par le renforcement des cibles, mais il se préoccupe avant tout d'influencer les comportements sociaux ${ }^{23}$. 》

Dans cet urbanisme affinitaire et défensif, tout l'arsenal du blindage est utilisé : enceinte, barbelés, pics, vidéosurveillance (obstacle), entrée contrôlée par des gardes privés ou des systèmes de codes (triage), etc. Les concepteurs

21. Wrever O., "Securitization and Desecuritization", in Lipschutz R.D. (ed.), On Security, New York, Columbia university Press, 1998, pp. 46-87.

22. Sur les enjeux de politique intérieure qui fondent la décision, voir note 10.

23. Blakely E.J., Snyder M.G., Fortress America. Gated Communities in the United States, Washington (DC)/Cambridge, Brookings Institution Press/Lincoln Institute of Land Policy, 1997, p. 162 (notre traduction). Voir aussi Caldeira T., « Un nouveau modèle de ségrégation spatiale : les murs de Sao Paulo ", Revue internationale des sciences sociales, $\mathrm{n}^{\circ} 147,1996$, pp. 65-77; Le Goix R., «Quartiers fermés, intérêts particuliers », Urbanisme, n³37, 2004. 
expriment ainsi la volonté de protéger personnes et biens de la «ville contaminée » et de «la société incivile des zones défavorisées » 24.

La technologie militaire est aussi utilisée dans le blindage des frontières entre Etats. Lorsque la politique sécuritaire américaine a établi la nécessité d'une barrière pour renforcer, à la frontière mexicaine, le contrôle des passages clandestins de migrants et de trafiquants, l'armée américaine a été présente dès le début, aux côtés de l'Immigration and Naturalization Service (INS) 25. Sous le prétexte de la lutte contre les narcotrafiquants, elle a notamment construit le premier tronçon du mur, au niveau de San Diego, en recyclant des panneaux métalliques utilisés pour les pistes d'atterrissage lors de la première guerre du Golfe. De nombreuses technologies expérimentées sur des théâtres d'opérations ont ensuite été reconverties dans la surveillance de la frontière, grâce aux travaux de bureaux d'études publics et privés spécialisés dans les questions de défense ${ }^{26}$. La crise engendrée par les attentats de 2001 n'a fait qu'accentuer cette convergence du civil et du militaire, dans le cadre du Homeland Security Act en 2002 et de la Secure Border Initiative en 2006 : le souci sécuritaire et la criminalisation de l'immigration s'y mêlent étroitement. Le Secure Fence Act d'octobre 2006 prévoit la construction d'un double mur de $1000 \mathrm{~km}$ le long de la frontière mexicaine (obstacle), ainsi que l'installation d'un puissant réseau de surveillance électronique sur toutes les frontières terrestres, SBInet 27 (triage). Le ministère de la Sécurité intérieure a entrepris de réaliser un "mur virtuel », constitué de moyens électroniques de surveillance permanente (1 800 tours de surveillance équipées de caméras à infrarouge et de radars ultrasensibles, capteurs nouvelle génération, drones, réseau satellitaire, etc.) (obstacle). Celui-ci est connecté aux bases de données qui permettent de trier les personnes aux différents ports of entry (triage).

On a assisté à la même reconversion technologique lorsqu'en Espagne, le gouvernement Aznar entreprit le blindage de sa frontière méridionale. En 19971998, les villes espagnoles en territoire marocain, Ceuta et Melilla, furent entourées de barrières défensives. Celles-ci ont été renforcées, après les assauts des

24. Donzelot J., "La nouvelle question urbaine», Esprit, n¹1, 1999, p. 106 ; Low S.M., "Construire l'exclusion à travers les communautés fermées ", Les Annales de la recherche urbaine, $\mathrm{n}^{\circ} 93,2003$, pp. 149-157. Il n'est pas possible ici de traiter du rôle (incontestablement incitateur) de la promotion immobilière, qui anticipe sur une demande de sécurité de la part des futurs acquéreurs et contribue ainsi à la diffusion de la demande de blindage.

25 . Au début des années 1990, application de la stratégie "Prevention throught deterrence " (prévention par la dissuasion) dans la surveillance de la frontière avec, notamment, l'opération Gatekeeper. Voir Nevins J., Operation Gatekeeper. The Rise of "Illegal Alien" and the Making of the U.S.-Mexico Boundary, New York, Londres, Routledge, 2002.

26 . Andreas P., Border Games, Policing the U.S.-Mexico Divide, Ithaca, Cornell University Press, 2000 ; Andreas P., Biersteker T.J. (eds.), The Rebordering of North America, New York, Londres, Routledge, 2003 ; Waller Meyers D., From Horseback to High-tech: US Border Enforcement, Migration Policy Institute, février 2006, http://www.migrationinformation.org

27. SBInet: Secure Border Initiative Network. 
clandestins en 2005, par un nouveau système anti-intrusion (double muraille, sécurisation des outils de détection, installation d'un réseau de câbles d'acier empêchant l'approche, système de diffusion de gaz lacrymogène). En 1998, l'Espagne a également instauré un système de surveillance de ses côtes, le SIVE, géré par la direction générale de la Guardia civile et considéré comme un véritable « rideau de fer technologique 28 ». En effet, peu à peu doté des technologies de pointe en la matière (système de détection par tours de surveillance équipées de radars, de caméras thermiques et à infrarouge, de capteurs sensoriels, installé le long du détroit de Gibraltar, de la côte andalouse, des Iles Canaries; système centralisé de communication des données par satellite), le SIVE commande, via son centre de contrôle, des unités d'intervention maritimes et aériennes. Egalement financé par l'Union européenne, il est la pièce maîtresse de la sécurisation de la Méditerranée, de la «mer blindée », que vient compléter la coopération entre Etats européens pour les interceptions en mer (opérations Ulysse, Triton, Neptune en 2002-2003), puis l'institutionnalisation de cette coopération avec la création de l'agence européenne FRONTEX en $2004{ }^{29}$. Cet ensemble fait fonction d'obstacle en blindant terre et mer. Toutes les politiques migratoires nationales et européenne (procédures de visas, quotas, contrôle à distance, accords bilatéraux, etc.) font fonction de triage.

Le security fence que l'Etat d'Israël construit le long et à l'intérieur du territoire occupé de Cisjordanie est exemplaire de cette technologie asymétrique. Elle avait déjà été appliquée lors de la première Intifada (1987-1993) pour contrôler la bande de Gaza en l'entourant d'une barrière électrifiée, équipée de détecteurs et longée par une route de patrouille ${ }^{30}$ (obstacle). A partir de la seconde Intifada (2000-2005), le gouvernement a estimé devoir étendre ce dispositif à la Cisjordanie, en le justifiant par le risque terroriste ${ }^{31}$. Il considère devoir protéger sa population en sécurisant les deux côtés de la ligne verte par une Seam Area, une zone militaire fermée ${ }^{32}$, la partie interne étant contrôlée par la police des frontières, la partie externe, en territoire occupé, par les forces armées israéliennes. A partir de 2002, il a été décidé d'établir une barrière permanente, susceptible d'empêcher tout passage non contrôlé de véhicule, et de créer la Seam Area Administration. La barrière, entièrement gérée par le ministère de la Défense et par l'armée, ne suit pas exactement le tracé de la ligne verte, mais s'enfonce souvent de plusieurs kilomètres en territoire cisjordanien (sa lon-

28. SIVE : Sistema integrado de vigilancia exterior.

29. FRONTEX : Agence européenne pour la gestion des frontières extérieures.

30. "Lessons of the Gaza Security Fence for the West Bank", Institute for Contemporary Affairs, 2004, citée par Backmann R., op. cit., p. 52. La barrière de Gaza s'inspire elle-même du blindage de la «bonne frontière » avec le Liban, à la fin des années 1970.

31. Voir le ministère de la Défense, http://www.seamzone.mod.gov.il

32. Littéralement, zone de couture, de jointure: en hébreu «kaf hatefer». "Dans ce pays, dans la langue des Maitres $d u$ checkpoint, cela désigne la ligne de contiguité et évoque le fil très fin utilisé pour raccommoder les vêtements. C'est la ligne qui désigne le rapiéçage, la découpure, le morcean de tissu que le fil à la fois répare et met en évidence. C'est le lien entre deux morceaux de tissu, la suture de deux lèvres d'une plaie. ", Bishara A., Checkpoint, Arles, Actes Sud, 2004, p. 20. 
gueur devrait être plus de deux fois celle de la ligne verte : $730 \mathrm{~km}$ au lieu de $315 \mathrm{~km}$ ). Il s'agit d'un dispositif de défense complexe (multi-layered fence system), articulé autour d'une barrière de détection électronique. Il consiste à l'est (en Cisjordanie) en une route de patrouille protégée par des barbelés et bordée sur l'extérieur par une tranchée ; à l'ouest (en Israël) en une route anti-intrusion (intrusion tracking dirt road), une route de patrouille, une route pour véhicules blindés, le tout bordé de barbelés; au total, le dispositif occupe entre 60 et 100 $\mathrm{m}$ de large. L'ensemble est placé sous surveillance électronique et vidéo grâce à des tours de surveillance et les données informatisées sont centralisées dans des centres de commandement opérationnel. On y a ajouté, en certains endroits, un mur de béton de huit mètres de haut, inspiré des protections anti-tirs (gunfire protections) que l'armée utilise depuis longtemps le long des autoroutes en Cisjordanie (obstacle). Les passages entre Israël et la Cisjordanie ne peuvent s'effectuer qu'aux checkpoints, contrôlés par l'armée, et pour certains progressivement transférés à des sociétés de sécurité privées ${ }^{33}$; le système des permis de circulation pour les Palestiniens a été systématisé (triage). Le Mur israélien est aussi composé, en Cisjordanie, d'un ensemble d'enclaves israéliennes fortifiées (dites « colonies ») ${ }^{34}$, et d'un réseau de communication réservé aux citoyens israéliens (autoroutes, ponts, tunnels), ainsi que de 630 checkpoints environ ${ }^{35}$. Il se dédouble ainsi en une véritable «matrice de contrôle 36 » à trois dimensions, séparant les populations et bloquant tout exercice de souveraineté palestinienne.

Sur ces bases s'est créé un nouveau secteur pour le marché mondial de la sécurité : marché hautement concurrentiel, que se partagent quelques entreprises spécialisées, générant un peu partout des effets d'importation et d'imitation, institutionnalisant encore plus ces pratiques de blindage de la contiguité. C'est ainsi que le blindage électronique des frontières états-uniennnes a été confié aux fournisseurs habituels du Pentagone, réunis en consortium autour de la société Boeing, parmi lesquels figure Kollsman, filiale de la société israélienne Elbit Systems (défense et sécurité), qui elle-même équipe le security fence israélien ${ }^{37}$. La technologie israélienne en la matière a aussi été exportée en Inde, pour le blindage des frontières ${ }^{38}$. Quant aux systèmes de surveillance sophistiqués mis en place sur les côtes méditerranéennes par les sociétés espagnoles Amper (défense et sécurité ; intégration de systèmes) et Indra (technologies de l'information), ils équipent aussi les frontières à l'est de l'Europe (Serbie, Monténégro,

33. Rapoport M., «La sécurité nationale, un nouveau créneau pour le business », Ha'Aretz, cité par Courrier international, n884, 11 octobre 2007.

34. Elles constituent des «zones de sécurité spéciales» fortifiées de "barrières en profondeur ", qui totalisent, quant à elles, $700 \mathrm{~km}$ de blindage.

35 . Source : OCHA, ONU, septembre 2008, http://ochaonline2.un.org

36. Halper J., The Matrix of Control, 2001, http://www.mediamonitors.net/halper2.html

37. Ktitareff M., «Les Etats-Unis inventent la frontière high-tech», Les Echos, 18 décembre 2006.

38 . Jaffrelot C., "Inde-Israël : le nouvel élément-clé de l'axe du Bien ? », Critique internationale, n'21, 2003, pp. 24-32 ; Ramachandran S., "India and Israel United in Defense", Asia Times on-line, juin 2002, http://www.atimes.com 
Estonie), celles des pays du Sud impliqués dans la politique européenne de voisinage, le blindage des frontières des Emirats arabes unis, etc.

Le Mur s'écrit ainsi au superlatif : des technologies toujours plus performantes, des murailles toujours plus hautes, des contrôles toujours plus sophistiqués, toujours plus de gardiens et de financements... Toujours plus : cette monumentalité, cette théâtralité sont les signes de sa qualité performative.

\section{"Les maîtres $d u$ checkpoint 39 » : un pouvoir asymétrique}

Ce déferlement technologique est politiquement légitimé par l'affirmation de la nécessité d'empêcher les intrusions : c'est une barrière. Cet acte de blindage fait fonctionner un système de triage des individus et de contrôle des mouvements : c'est un ensemble de points de passage, de checkpoints. La barrière n'est qu'un des éléments architecturaux (avec le checkpoint, la zone de transit, le camp de rétention, la prison) d'un dispositif de sécurisation complexe, au sens foucaldien du terme 40 : celui-ci se compose aussi de technologies complexes, de pratiques de contrôle et de surveillance policières ou privées, de réglementations et de législations sur l'accès au territoire, de discours professionnels et politiques sur la securitization de la limite territoriale, etc. Sur le plan pratique, la barrière est loin d'être essentielle, ne serait-ce que parce qu'elle peut être contournée de mille manières (tunnels, franchissements par effraction, passages frauduleux aux checkpoints, modifications des trajets, etc.). La barrière a une simple fonction d'obstacle : ce qui importe, dans le Mur, c'est le checkpoint, qui signifie arrêt et contrôle ; en assurant l'interconnexion des différents processus de surveillance, c'est le checkpoint, bien plus que la barrière, qui fait exister le pouvoir de contrôle. Ce pouvoir de contrôle s'exerce sur les déplacements des personnes : il limite arbitrairement, et sans recours possible, la liberté de circulation de certains, en fonction de leur statut ${ }^{41}$.

39. Bishara A., op. cit.

40. "Ce que j'essaie de repérer sous ce nom (dispositif) c'est, premièrement, un ensemble résolument bétérogène, comportant des discours, des institutions, des aménagements architecturaux, des décisions réglementaires, des lois [...]. Le dispositif lui-même, c'est le réseau qu'on peut établir entres ces éléments. Deuxièmement, ce que je voudrais repérer dans le dispositif, c'est justement la nature du lien qui peut exister entre ces éléments hétérogènes [...]. Troisièmement, par dispositif j'entends une sorte de formation qui, à un moment donné, a eu pour fonction majeure de répondre à une urgence. Le dispositif a donc une fonction stratégique dominante. ", Foucault M., Dits et écrits, t. 2, op. cit., p. 299.

41. La belle métaphore littéraire d'America, le roman de T.C. Boyle sur les gated communities et sur le rejet de l'étranger, l'illustre idéalement : "Non eux, c'étaient les humains qui les inquiétaient. Les Salvadoriens, les Mexicains, les Noirs, les tueurs des gangs, les taggers et autres voleurs de voitures dont ils lisaient les exploits dans le journal [...]. Non, sans arrêt, sur tous les tons et comme si c'était la clé de voûte de leur existence toute entière, ses voisins ne parlaient que d'une chose : les portails. De fait, ils ne parlaient même que d'un seul-celui qu'on allait bientôt ériger à l'entrée principale. Celui que vingt-quatre beures sur vingt-quatre un gardien surveillerait afin d'interdire l'accès des Domaines à tous ces taggers, tueurs des gangs et autres voleurs de voitures qu'ils étaient montés jusqu'ici pour fuir à jamais ", Boyle T.C., America, Paris, Grasset, 1997, p. 48. 
Le Mur est à la fois biface et bifonctionnel. La barrière est un élément de dissuasion : pour l'extérieur, elle veut signifier la difficulté et le danger à la franchir illégalement; pour l'intérieur, elle veut définir l'espace de protection et de sûreté que le pouvoir localisé prétend assurer à la communauté territorialisée ${ }^{42}$. La barrière est l'élément le plus visible et le plus monumental possible : c'est l'aspect performatif le plus important du dispositif.

\section{Performatif pour la gated community:}

«Le besoin d'efficacité de ces portails efface tout prestige : cônes, pics, herses, barrières, chicanes, etc., se dressent sans gêne dans l'espace public. Les connotations militaires de l'architecture de ce dispositif de filtrage ne sont pas euphémisées. Bien au contraire, cette esthétique défensive a une fonction. Elle rend visible la sécurité et le contrôle retrouvés de cette communauté ${ }^{43}$. »

\section{Performatif pour le security fence israélien :}

«Les murs autour des “îlots israéliens”, où s'applique la loi israélienne, sont conçus pour protéger les colons et exclure un extérieur effrayant. Les portes des barrières ouvrent sur des axes de circulation protégés, qui intègrent économiquement et politiquement les colons à Isräl. Les barrières, murs, fossés, levées, et autres sortes d'inventions territoriales placées autour des îlots palestiniens, sont conçus pour empêcher des "risques sécuritaires" de s'en échapper. En redessinant et en contraignant l'habitat, en marquant physiquement les limites des différentes juridictions, ces barrières fonctionnent surtout comme une machine de contrôle de la population. Plus qu'un simple système de fortification, elles deviennent un mécanisme bureaucratique et logistique de création et de maintien de la séparation démographique ${ }^{44}$. »

42 . "Depuis que l'insécurité individuelle s'enracine en des lieux anonymes, lointains et inaccessibles, on ne voit pas très bien ce que les pouvoirs locaux, visibles, pourraient faire pour alléger ces troubles; par contre il semble y avoir là une réponse évidente et directe à cet autre malaise, celui qui concerne l'identité collective : les pouvoirs étatiques territorialisés peuvent encore être utilisés pour fermer les frontières aux migrants, limiter les droits d'asile, rafler et déporter les étrangers indésirables. Les gouvernements ne peuvent pas honnêtement promettre à leurs citoyens une existence sûre et un futur assuré; mais ils peuvent un temps les décharger en partie de leur anxiété (et éventuellement en profiter sur le plan électoral) en affichant leur énergie et leur détermination dans la guerre contre les demandeurs d'emploi étrangers et autres gate-crashers, intrus dans l'arrière cour autochtone, jusqu'ici propre et tranquille, ordonnée et familière ", Bauman Z., Europe of Strangers, Working paper, 2002, pp. 10-11, http ://www.transcomm.ox.ac.uk (notre traduction).

43. Corompt G., «Des limites révélatrices : l'œil de l'architecte sur les communautés fermées ", cité par Billard G., Chevallier J., Madoré F., Ville fermée, ville surveillée. La sécurisation des espaces résidentiels en France et en Amérique du Nord, Rennes, Presses universitaires de Rennes, 2005, p. 125. Cet étalage sécuritaire n'est le fait que de certaines gated communities. D'autres, à l'inverse, dissimulent leurs techniques de blindage.

44. Weizman E., Hollow Land. Israel's Architecture of Occupation, New York/Londres, Verso, 2007, p. 178 (notre traduction); voir aussi Weizman E., Segal R. (eds.), Une occupation civile. 
D’un point de vue architectural, la barrière fait exister, malgré le démenti de la globalisation, un espace intérieur et un espace extérieur : cette incarnation fantasmatique est lourde de sens politique. Elle dit un espace de la sécurité et un espace du risque. L'espace de la sécurité est créé et protégé au nom d'une identité partagée, fondatrice d'une communauté. L'espace du risque est compris comme celui de l'altérité et des catégories stigmatisées : «une villa dans la jungle » dit-on d'Israël ${ }^{45}$. Ainsi, pour la gated community:

« la fermeture ajoute à l'agrégation, la rend plus manifeste, tend désormais à faire considérer à ceux de l'extérieur qu'une limite sociétale infranchissable existe en même temps qu'elle oblige ceux de l'intérieur à se sentir nécessairement plus solidaires ${ }^{46}$. »

De même, le blindage de la frontière entre l'Inde et le Pakistan est-il l'occasion de virulentes démonstrations nationalistes des deux côtés, devenues quasispectacles, lors de la relève de la garde à l'unique poste frontière de Wagha ${ }^{47}$.

Le checkpoint, quant à lui, est un élément de triage et d'éventuelle répression : pour les entrées, il sélectionne les bienvenus et rejette les indésirables; pour l'intérieur, il assure une sûreté liée au statut, celle que semble garantir l'entre-soi. Cet élément, constitué de l'interconnexion des systèmes d'information, est le plus discret et le plus sophistiqué : il tient son efficacité de sa capacité technologique à distinguer les statuts. En effet, l'histoire du Mur est une histoire de statuts, de frontière de statuts. Du côté intérieur, le statut est reconnu et assure la liberté de circulation. De l'autre côté, le statut est incertain et la liberté de circulation restreinte. Le tri entre les statuts se fait au checkpoint.

«Le checkpoint représente un point de transformation dans l'espace public; ce n'est pas un lieu; c'est une bordure, une frontière, un point de passage, un début et une fin en ce même point de l'espace public. C'est un déplacement au bord de l'abîme. [...] C'est un lieu de domination et de despotisme. C'est un site et une fortification, visibles uniquement de l'extérieur. Le voir est permis, le traverser ou le toucher est prohibé. Ce n'est pas un espace public. C'est un espace qui contrôle l'espace public ${ }^{48}$. »

La politique de l'architecture israélienne, Paris, Editions de l'Imprimeur, 2004.

45. Ehoud Barak, ex-Premier ministre d'Israël, cité par Cypel S., Les Emmurés. La société israélienne dans l'impasse, Paris, La Découverte, 2005, p. 85.

46. Billard G., Chevalier J., Madoré F., op. cit., p. 82.

47. Voir l'illustration iconographique donnée dans : Novosseloff A., Neisse F., Des murs entre les hommes, Paris, La Documentation française, 2007.

48. Bishara A., op. cit., pp. 26-27. 


\section{«Les enfermés dehors » : l'asymétrie comme humiliation}

En ce sens, le Mur n'est qu'une des modalités, certes une des plus visibles, de la société de contrôle. A le regarder trop rapidement, il donne l'impression de re-territorialiser les contrôles, sur un mode quelque peu archaïque. En réalité, en tant que pièce d'un dispositif de contrôle, il participe de la diversification et de la dissémination des limites (statutaires) dans un monde-frontière.

«Dans ce monde-frontière parcouru par d'innombrables flux constitués (au-delà et bien avant les mouvements de marchandises, de capitaux et de personnes circulantes) par les décisions prises ou imposées, par les politiques adoptées, par les influences exercées par les divers acteurs globaux - les frontières constituées par les statuts (économiques, sociaux, juridiques) apparaissent toujours plus comme les éléments (comme les frontières) qui permettent, entravent, ou empêchent l'accès au même réseau de flux. Les individus représentent en ce sens des manifestations éparses des frontières constituées par les statuts dont ils sont titulaires ${ }^{49}$. »

Dans ce monde-frontière, la capacité de mobilité est un élément déterminant de la stratification sociale globale. Dans cette nouvelle hiérarchie, les plus libres sont protégés par le Mur : les cosmopolites, l'élite globale mobile, qui se déplacent de sanctuaire en sanctuaire (eux-mêmes blindés), selon des trajets réservés (eux aussi blindés), et les migrants réguliers que cette élite autorise à pénétrer sur ses territoires. D’autres, tout en pratiquant la mobilité, le font illégalement : ceux-là, les clandestins, le Mur ne les arrête pas. Peut-être même estil là pour les maintenir dans la catégorie des sans (pour parler comme Hannah Arendt ${ }^{50}$ ), sans papiers / sans statut. C’est ainsi par exemple que :

« la militarisation anti-migratoire des rives sud et est de la Méditerranée semble destinée à assurer plutôt la subordination des étrangers - leur entrée et leur maintien comme métèques ou invités invisibles, sous payés et sans droits -, que leur exclusion préventive. C’est vers la marginalisation interne, plus que vers le refus, que semble orientée la militarisation des frontières méridionales du monde riche 51 ».

49. Cuttitta P., «Le monde-frontière. Le contrôle de l'immigration dans l'espace globalisé ", Cultures E Conflits, $\mathrm{n}^{\circ} 68$, hiver 2007, http://www.conflits.org/index5593.html ; Z. Bauman théorise lui aussi le "pays de la frontière " : "l'espace global a adopté le caractère d'un pays de la frontière ", dans La Société assiégée, op. cit., p. 129 ; voir également Kalm S., Migration Control Policies as Spatial Organization. Mobility, Power and Geopolitical Imaginations, AMID Working Paper Series 39/2005, http://www.amid.dk/pub/index.html\#top

50 . Arendt H., L'Impérialisme, Paris, Fayard, 1982.

51. Dal Lago A., 2005, op. cit., p. 13 (notre traduction). 
Il y a, en effet, pour la plupart des économies développées, une utilité fonctionnelle à conserver un volant de main-d'œuvre clandestine, qui permet de maintenir la flexibilité du marché de l'emploi. Ce maintien dans la marginalité économique et sociale induit, en un cercle vicieux bien connu, la stigmatisation des exclus sous la forme de la criminalisation des outsiders : la frontière statutaire est pour eux toujours incertaine et souvent marquée d'allers-retours entre légalité et illégalité, au gré du marché du travail et de la loi migratoire ${ }^{52}$. Toutes les réactions des Latino-Américains aux Etats-Unis, face à la criminalisation de l'immigration qui a entraîné la construction de la border fence en attestent : en arrêtant durant une journée toute activité économique, ils ont démontré la fonctionnalité de leur présence pour le marché états-unien ${ }^{53}$.

Enfin, les confinés, les indésirables absolus, sont cloués au sol par les règlements et les contrôles à distance : ceux-ci ne s'approchent même pas du Mur et restent bloqués dans les «non-zones » 54 . Peut-être faut-il ajouter à cette nouvelle stratification les transitaires, tous ceux qui font commerce de la dissymétrie économique (activités de maquilladoras, économies des zones frontières) et du passage de l'obstacle territorialisé (activités de passeurs), dont les intérêts fluctuent avec le degré de fermeture du Mur et la plasticité des statuts. Le Mur souligne ainsi la qualité de "membrane asymétrique 55 » des limites territoriales et statutaires contemporaines : permettant les sorties et contrôlant l'accès, laissant circuler les capitaux et restreignant les mouvements des travailleurs (Peter Andreas écrit à juste titre, à propos de la frontière entre Etats-Unis et Mexique, «économie sans frontières, frontière blindée 56 »), protégeant la communauté interne et réprimant les outsiders, confrontant richesse et pauvreté. La stratification s'opère sur l'inégalité des droits à la mobilité et sur la détention du pouvoir de contrôler les déplacements. Qui contrôle l'accès - la possibilité d'accès - exerce le pouvoir : il est le «maître du checkpoint ", celui qui s'autorise à catégoriser, à hiérarchiser des statuts. Le Mur fait exister, fait s'incarner les catégories qui le légitiment.

52. Calavita K., Immigrants at the Margins. Law, Race, and Exclusion in Southern Europe, Cambridge, Cambridge University Press, 2005.

53. La «Gran marcha» et le «Jour sans immigrants» de 2006. Voir Richard E., « Aux EtatsUnis, les clandestins sont exploités », Libération, 2 mai 2006 ; Mulard C., " Les Latinos aux Etats-Unis, sûrs de leur force », Le Monde, 20 avril 2006.

54. Le terme "remote control " (contrôle à distance) est utilisé dans : Zolberg A., "Matters of State: Theorizing Immigration Policy”, in Hirschman C., Kasinitz P., De Wind J. (eds.), The Handbook of International Migration: The American Experience, New York, Russell Sage, 1999 ; celui de «non-zones » dans : Rufin J.-C., Globalia, Paris, Gallimard, 2004, notamment p. 331.

55. Hedetoft U., The Global Turn: National Encounters with the World, Aalborg, Aalborg University Press, 2003.

56. "Borderless economy, barricaded border", Andreas P., “The Wall after the Wall”, in Andreas P., Snyder T. (eds.), The Wall around the West. State Borders and Immigration Controls in North America and Europe, Laham, Oxford, Rowman \& Littlefield Publishers, 2000 , p. 4. 
L'histoire du Mur est une histoire d'accès, contrôle de l'accès, inégalités d'accès : il faut avoir le mot de passe, le code, les moyens financiers, le «bon » statut, etc., ou bien transgresser l'ordre établi par le Mur. Les différentes voies d'accès aux checkpoints (postes frontières, postes de contrôle, ports of entry, entrée unique des résidences fermées) en sont l'illustration parfaite, avec une différence d'aisance dans le passage selon le statut des individus : plus le statut est reconnu, plus les moyens d'en attester sont sophistiqués (informatique et biométrie), plus aisé sera le passage (par exemple pour les détenteurs du passeport Schengen et des passeports biométriques), jusqu'à être parfois automatisé (sur les autoroutes reliant Etats-Unis et Mexique par exemple, pour les ressortissants américains effectuant quotidiennement l'aller-retour). Pour les autres, à l'inverse, les files d'attente s'allongent et les vexations se multiplient. Ces effets frontières, parce que statutaires, se démultiplient, se « pixellisent ", avec les déplacements des personnes : les conditions inégalitaires d'accès au checkpoint sont aussi présentes, en miroir, dans les pratiques administratives routinisées (dans les files d'attente devant les consulats, dans les files aux guichets des préfectures, dans les zones d'attente des aéroports, etc. ${ }^{57}$ ). Le dispositif du Mur, frontière de statuts est, en fait, un archipel de contrôles.

La matérialisation de ces inégalités statutaires, outre son effet pratique de limitation de la liberté de certains, a aussi un effet symbolique, celui de l'humiliation. La monumentalité de la barrière, qui fait réassurance et identité pour les insiders, est aussi profondément humiliante pour les outsiders. Le soupçon qu'incarne le pesant blindage de la séparation, l'acceptation passive des files d'attente, l'obligatoire soumission aux checkpoints, le fréquent arbitraire des contrôles, les procédures de refus d'entrée ou d'expulsion sont autant de mises en scène de la domination. Ce sentiment d'humiliation est très difficile à restituer, sans une expérience sensible directe : ce n'est pas un hasard si les photographes, les vidéastes, les architectes et les plasticiens se sont beaucoup investis dans la contestation du Mur. Leurs interventions sont devenues de véritables ressources pour toutes les campagnes «Stop the Wall» qui se répondent de par le monde, de la Palestine au Mexique, des Territoires sahraouis à Padoue. C'est aussi cette puissance symbolique qu'exprime l'emploi généralisé du terme «mur » pour un dispositif bien plus vaste et plus complexe qu'une simple barrière : «mur » fait image, de protection ou d'enfermement, de sûreté ou d'humiliation, c'est selon.

Aussi, pour évoquer cette violence matérielle et symbolique, peut-être vaut-il mieux, in fine, écouter les paroles d'un témoin direct :

«Chez tous les perclus de la clôture, reclus ou exclus, elle fait de l'emmuré un obsessionnel et de l'emmurant un paranoïaque. 
Pourrais-je passer ? Et si je tombe sur un barrage volant ? A quelle heure ferment-ils ? Par où prendre aujourd'hui ? Et comment faire pour revenir ? Ce n'est pas trop tard ? Tout protégé que je sois par mon passeport d'étranger de ces réflexes conditionnés de rat pris dans un labyrinthe, je me sens déjà rongé par l'anxiété du sésame de la bonne couleur, de la fermeture sans préavis, de la bonne ou mauvaise plaque d'immatriculation... 58 "

58. Debray R., Un candide en Terre sainte, Paris, Gallimard, p. 400. L'humiliation est aussi le sujet du livre de Bishara A., op. cit. 